\title{
GrabApple: The Design of a Casual Exergame
}

\author{
Yue Gao and Regan L. Mandryk \\ Department of Computer Science, University of Saskatchewan, \\ Saskatoon, Saskatchewan, Canada \\ \{yue.gao, regan.mandryk\}@usask.ca
}

\begin{abstract}
Many people do not get the recommended 30 minutes of exercise per day, which can result in health problems such as obesity, muscle atrophy and chronic disease. Based on the principles of casual games and exergames, we propose and define casual exergames for motivating people to exercise in multiple small chunks of time throughout the day. We designed, implemented, and tested a casual exergame called GrabApple. Our preliminary results show that users enjoyed playing the casual exergame and that in just 10 minutes of play, their heart rate was elevated to an average of $72 \%$ of maximum heart rate, and that they burned an average of 91.8 Calories. If played three times per day, our casual exergame produced sufficient physical activity to meet current fitness guidelines. We discuss the potential health benefits of casual exergames.
\end{abstract}

Keywords: Exercise, casual game, exergame, exercise efficacy.

\section{Introduction}

People should do at least 30 minutes of moderate-intensity exercise a day; however, most people do not perform this suggested amount of daily exercise despite the benefits [1]. First, regular exercise can reduce the risks of developing coronary heart disease, hypertension, high blood pressure, colon cancer, obesity, and diabetes [1]. Second, physical activity can relieve symptoms of depression and anxiety, and improve mood [1]. Third, aerobic exercise has been linked to cognitive benefits, improving many aspects of cognition and brain function [2]. Finally, a healthy society produces less strain on an overburdened health care system.

One strategy to encourage people to get their recommended amount of daily activity is to break the 30 minutes of exercise into smaller chunks so it is more manageable. People might then be able to better manage this time commitment, and there would also be a lower overhead to exercising, including reducing the need to change into exercise clothes or shower after exercising. Research supports that breaking exercise into small chunks is a good approach because the physical benefits of three moderate-intensity short bouts of physical activity (lasting about 10 minutes) are similar to one continuous 30-minute bout $[3,4]$. Still, given a 10-minute break in their day, many people do not choose to use this time to exercise because typical short bursts of activity, such as climbing stairs or jogging for 10 minutes are not very enjoyable. Many people say they do not exercise so they can do other activities, such as watching television (47\% of respondents selected this), sleeping in (43\%), doing household chores $(59 \%)$ or working $(43 \%)$ [5]. 
One approach to making exercise more fun is through the use of exergames, which are games that use exercise as part of the activity required to play a video game $[6,7]$. Exergames have been shown to be more fun to play than a corresponding level of standard exercise [7]. Although exergames can promote physical activity, these games are often tedious to set up, use specialized hardware, and need a committed chunk of time to play. To motivate people to do 10-minute bouts of exercise in a fun way, we take the novel approach of applying the principles of casual games - which have been described as games that are "fun, quick to access, easy to learn and require no previous special video game skills, expertise or regular time commitment to play" ([8], p.3) - to exergames [7], creating the new genre of casual exergames. We define casual exergames as games that players can learn easily and access quickly, using simple rules and special game mechanics, to motivate them to exercise at a moderate intensity for short periods of play. The advantages of casual exergames are two-fold. First, casual games appeal to people of all ages, genders and nationalities [8], are fun, and are easy to start and stop playing. Second, exergames have game mechanics promoting exercise so it makes exercise seem less difficult and more enjoyable [7]. Therefore, casual exergames should motivate physical play in small manageable chunks of time, and help people to exercise for the recommended 30 minutes per day.

We designed and implemented a casual exergame based on the principles of casual games and exergames. To make the game simple to play we use the player's body as the game controller, where movement is sensed through the use of inexpensive and accessible hardware (Microsoft Kinect sensor). Through a four-month development process, we iteratively playtested our game, generating a short, fun, and easy-to-learn play experience that caused players to elevate their heart rate and burn Calories.

We then conducted a preliminary study to investigate how much fun our game is and how much exercise it produces in players. We compared our casual exergame to a mouse-based version of the game using eight study participants. Initial results showed that the casual exergame is fun to play and can elevate players' heart rates to target levels for moderate-intensity aerobic exercise. We discuss the potential health benefits of casual exergames and present future research opportunities in this space.

\section{Defining Casual Exergames}

\subsection{Casual Games}

In 2006, the casual games industry was a $\$ 2.25$ billion/year industry and was growing by $20 \% / y e a r$ [8]. This was prior to the smartphone and tablet revolution, which has catapulted casual games into an entirely new level of popularity. There are many definitions for casual games and the community has yet to converge on a final definition [9]. The Casual Games SIG of the International Game Developer's Association (IGDA) defines casual games as "games with a low barrier to entry that can be enjoyed in short increments" ([10], p.9). The casual games community has generally agreed on the following criteria for a game to be considered casual:

Easy to learn. Casual games should have limited instructions, provide rules that are easy to learn, and guide players with a clear and consistent user interface [10]. 
Simple controls. Casual games should take advantage of assumed knowledge, such as drag, drop and click [10].

Play in a relatively short play period. Casual games should be able to be enjoyed in a series of short time increments, though sometimes people play one level after another for many hours [8]. Most casual games' play intervals are between 5 and 15 minutes to complete a level [8].

Reduced complexity and non-punishing game play. Casual games allow depth and complexity to emerge from the player's basic actions instead of making the player master a large and complex set of actions [10].

Family friendly. The IGDA Casual Games SIG deems that casual games should not contain objectionable content, such as overt violence, or sexuality [10]. The CGA agrees that casual games should appeal to people of all ages, genders and nationalities [8]. In recent years, casual games have been developed (e.g., games rated 17+ for Apple's iPad) that do not adhere to this criterion.

\subsection{Exergames}

Exergames can motivate players to be more physically active by combining games and exercise together. Some commercial games can be considered exergames. For example, in Dance-Dance Revolution, players step on colored sections on a platform to musical and visual cues [11]. Wii Fit works directly with the Wii console to bring players a fun and exciting way to experience fitness [12].

Researchers have designed and tested a variety of exergames. Some games use custom sensors such as in Ping Pong Plus [13], Breakout for Two [6] and Kick-Ass Kung $\mathrm{Fu}$ [14]. Others have integrated standard exercise equipment, such as the stationary bikes used in Heart Burn [15] and Life is a Village [16]. Exergames have also been developed that do not prescribe the type of exercise, but are based on the player's heart rate, such as Triple Beat [17] and Nenonen et al.'s biathlon game [18].

Because our game is intended to be a casual game, the overhead to play needs to be minimal and we cannot use expensive hardware, such as resistance bikes or custom sensor floors. We chose to use the movement of the body as input to the game, requiring no game controller or exercise equipment.

Efficacy of Exergames. The goal of exergames is to create play experiences that are both fun and that elevate heart rate. In general, researchers have shown that their exergames are fun to play, but do they have the same benefits as regular exercise? Research shows that many exergames can be utilized as part of an overall aerobic exercise program because in a 30-minute exercise session, a player's heart rate and caloric expenditure are both within the American College of Sports Medicine recommendation for daily physical activity [19]. However, in another study that investigated the relative efficacy of Wii games and their real life sports counterparts, it was shown that the energy that players used to play active Wii Sports games was not of high enough intensity to reach the recommended daily amount of exercise in 
children [20]. It is important for exergame researchers to demonstrate the efficacy of the exercise generated by their game to ensure that the players of these games are getting the health benefits of exercise. An alternative viewpoint is that playing exergames (as opposed to traditional games) means that players are at least unable to simultaneously play and work their way through an unhealthy snack such as a bag of potato chips. Although perhaps not a direct benefit, replacing traditional games with exergames will at the very least prevent this type of unhealthy behavior.

\subsection{Casual Exergames}

Because we are proposing a new genre of game, there is not a previous framework to understand the casual and exergame aspects of casual exergames. We propose that casual exergames are games that require exertion to play and adhere to the criteria of casual game design. More specifically we define casual games as games that players can learn easily and access quickly, using simple rules and special game mechanics, to motivate them to exercise at a moderate intensity for short periods of play.

Some people argue that exergames developed for recent commercial gaming platforms, such as Wii Sports, can be considered as casual games $[8,10]$ because they are easy to learn, are family friendly and use simple controls. However, many commercial exergames do not provide enough exercise intensity to be considered a casual exergame by our standards. For example, previous work shows that the energy used playing active Wii Sports games is not intense enough to reach the recommended daily amount of exercise for children [20]. In addition, these commercial games do not necessarily meet the criteria for casual games defined previously; i.e. casual games must be able to be played in a relatively short play period. If casual games are to be played in short play periods, they should also be quickly accessible games with little-to-no setup time [10]. If setting up a console game requires even 5 minutes to boot the system, navigate the menus, and calibrate the controllers, then it is not in line with the duration of play expected of a casual game. Finally, the accessibility of gaming consoles, which are generally kept in domestic environments, limits their use as systems on which to play exergames during breaks in the work day.

\section{GrabApple: A Casual Exergame}

We designed GrabApple-a casual exergame that uses the player's body as the game controller and the player's own body weight as resistance to elevate heart rate. In this section, we describe the design and implementation of GrabApple and show how it conforms to the principles of casual game design and efficacious exergame design.

\subsection{Game Concept}

In GrabApple (Figure 1), the goal is for players to try to pick up falling red and green apples and avoid touching the falling bombs. Apples and bombs are picked and 
touched using a virtual hand whose position on the screen is controlled through the movement of the player's body. Each apple picked adds one point to the total player score, while each bomb touched subtracts five points.

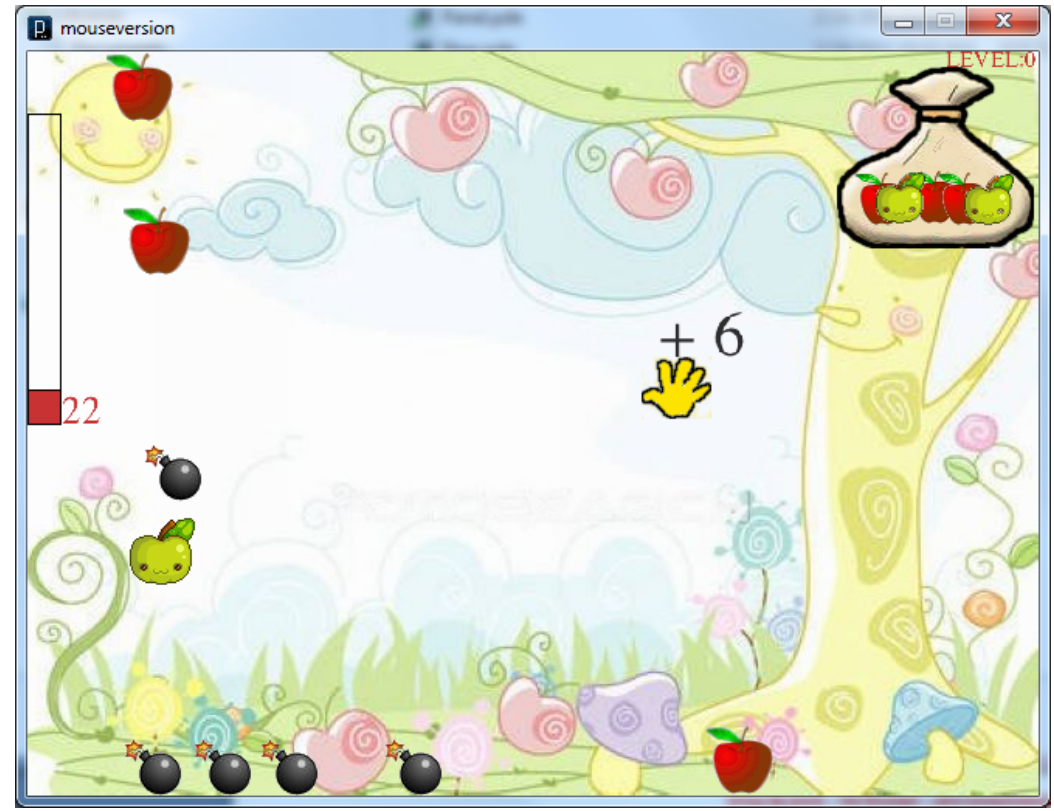

Fig. 1. GrabApple. Players move the hand to grab falling apples while avoiding falling bombs

Designing for Casual Interaction. We designed the game to conform to the principles of casual game design in the following ways:

1) Easy to learn. The concept of GrabApple is easy to learn - a player should touch the apples and avoid the bombs. There are only two additional mechanics: players can only hold five apples at a time and thus need to empty the virtual hand into a collection bag; and there is a mushroom bonus that appears and players have to press a key on the keyboard to get extra points. Also, we clearly provide feedback to players on their performance through floating scores and an energy bar that displays total points (Figure 1).

2) Simple controls. GrabApple uses the player's body as the game controller. The virtual hand is mapped to the player's movements so players jump, duck and move to control the virtual hand movement.

3) Play in a relatively short play period. GrabApple takes an average of three minutes to complete a game level. The setup time is also in line with short play periods. After plugging the Microsoft Kinect sensor into the computer's USB port, the player launches the application in a similar manner to most casual games. With the short setup time and the use of standard computers, players can quickly access and play our casual exergame. 
4) Reduced complexity and non-punishing game play. The game mechanics and controls of GrabApple are simple. In addition, it is difficult to lose the game, but players are rewarded after completing a level with an unlocked higher level and can compete for the high score.

5) Family friendly. GrabApple has no objectionable, violent, or sexual content.

Designing for Efficacious Exercise. We motivate people to get the recommended aerobic exercise intensity level through the game design and game mechanics.

1) We encourage players to move around. We give score multipliers for picking consecutive apple of the same color and give a penalty for touching any bombs. This encourages the players to move the virtual hand around the screen instead of standing and waiting for the apples to fall.

2) We elevate players' heart rates by using their own weight as resistance. The virtual hand on the screen can only hold five apples. Players have to empty the virtual hand by putting it into the collection bag on the right side of the screen. They can only reach this bag by running to the side and jumping, thus using their body as resistance and increasing their heart rate. Also, in order to pick up the maximum number of apples, players need to jump up and bend down.

3) We elevate players' heart rates by adding extra exercise. Once in a while, a mushroom appears on the screen and players can get a five-point bonus by picking up the mushroom. In order to receive the bonus points, players have to run to the keyboard and press any key. Players have to hurry, as the mushroom is only available for two seconds, so they need to reach the keyboard as fast as they can. Because players stand approximately 1.5 meters from the keyboard when playing the game, quickly running to the keyboard increases overall activity.

4) We raise exercise intensity by increasing game difficulty through game levels. In higher game levels, we increase the speed and the number of falling apples and bombs. These game levels become accessible when players get sufficient points as shown by the energy bar on the left of Figure 1.

We designed our game for casual play and for exercise, but also designed the game to be fun to play through the use of reward animations and player achievements [21].

\subsection{Game Implementation}

We implemented two versions of our game: the exergame and the mouse version. Both games were programmed in Processing. For the casual exergame, we use the Microsoft Kinect sensor to detect users' body movements. The Kinect is essentially comprised of one camera that detects $\mathrm{x}$ and $\mathrm{y}$ position and another infrared receiver camera that detects depth through the dispersion of dots displayed via an infrared transmitter. In our game, we can track the player's body movement by the average location of a given number of points that meet a specific depth threshold. We use Daniel Shiffman's Kinect library for Processing [22]. For the mouse-based version, we use the mouse cursor location to control the position of the hand on the screen and players left clicked the mouse to empty the hand into the collection bag. 


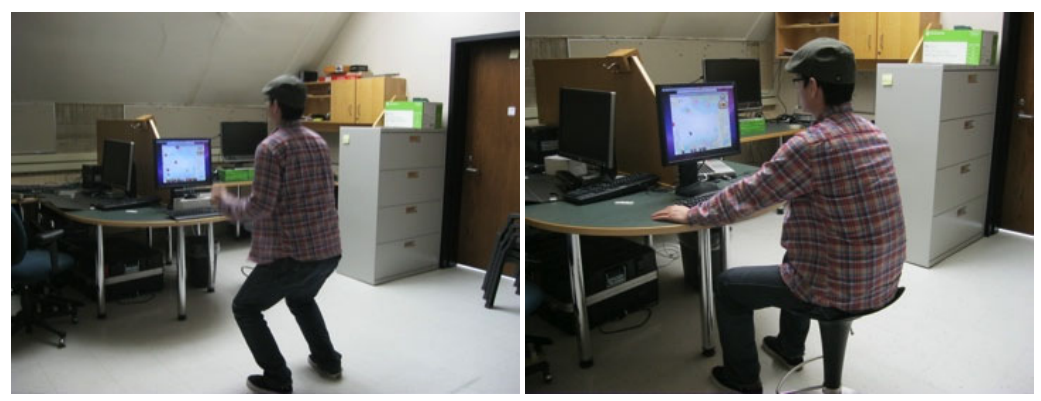

Fig. 2. Playing the Kinect (left) and mouse version (right)

\section{Preliminary Study}

We investigated the enjoyment of our game and the intensity of exercise during play.

\subsection{Participants}

We recruited 8 university students ( 7 male), aged 22 to 44 (mean of 29). Six of the participants played video games at least weekly; the other two played only a few times a year. Our players reported that they play different types of video games, including role-playing games, first-person shooters and puzzle games. None of our participants play games using the Kinect on a regular basis.

When asked about their daily exercise, only 3 out of 8 participants got 30 minutes of sustained physical activity per day. Four participants exercised for 15-30 minutes per day, while one exercised for 0-15 minutes per day. Most players (five) said that they do not have time to complete the daily-recommended amount of exercise, and three said that their lack of exercise was due to laziness. When asked if they take regular breaks during the workday, six of our players said that they take a break every hour or two. The other two reported that they seldom took breaks.

\subsection{Apparatus}

The game was played on Mac OS X 10.6.6 with a 20-inch monitor. A low resolution $(800 \times 600)$ was used as players stood about 1.5 meters from the display to play the casual exergame. During the play of the casual exergame, players wore a Garmin Forerunner 110 heart rate monitor with a strap around the chest so we could log their heart activity and the Calories burned.

\subsection{Procedure}

A within-subjects design was used where participants played both the casual exergame and the mouse-based version. To reduce crossover effects between conditions, players visited the laboratory on two consecutive days to participate. To reduce differences between conditions, we had participants play at approximately the same time each day and asked them to try to keep their patterns the same prior to each 
experimental session in terms of the amount and time of food and caffeine consumption, and the amount of sleep. To reduce order effects, half of the participants played the casual exergame on the first day, while the other half played the mousebased version first.

Participants began by filling out an informed consent form and learning the rules and procedures of the game through an initial training session. Then participants played the game for 10 minutes. After playing the game, participants rated their exertion level according to the Borg Rating of Perceived Exertion Scale, where people subjectively rate how hard they feel they are working on a 15 -point scale ranging from 6 (no exertion) to 20 (maximal exertion) [23].

After completing the second experimental session, participants filled out a questionnaire asking about their demographic information and for a comparison of the two game versions.

\section{Results}

We first present the results for the efficacy of exercise, followed by the results related to the enjoyment of the game.

\subsection{Efficacy of Exercise of GrabApple}

The American College of Sports Medicine recommendations for moderate-intensity exercise is $64-76 \%$ of maximum heart rate (220-age) [24]. After our participants played the casual exergame for 10 minutes, their average heart rate was $72 \%$ $(\mathrm{SD}=12 \%)$ of their maximum heart rate, which is in the range of moderate-intensity exercise. The average Calories burned in those 10 minutes was 91.8 ( $\mathrm{SD}=31.86)$. If we assume that participants play our game for three 10-minute bursts per day, the average Calorie expenditure will be 275, which exceeds the recommended Calories per day that adults should burn through aerobic exercise (200 Calories)[25]. The average Borg Rating of Perceived Exertion after playing the casual exergames was $12.38(\mathrm{SD}=0.85)$, which is within the recommended intensity for improving aerobic capacity of 12-13 (somewhat hard) to 15-16 (hard) [23]. The exercise efficacy of GrabApple as indicated by our measures is shown in Figure 3.

\subsection{Exergame vs. Mouse-Based Game}

We asked participants to rate five aspects of the game (fun, exciting, challenging, frustrating, and easy to learn) on a 5-point Likert scale (1=strongly disagree, $5=$ strongly agree) after playing each version. Given the non-parametric nature of our ratings data, we transformed these ratings into ranks - for each question, participants chose the mouse version, the Kinect version, or neither. Figure 4 shows the rankings. A chi-squared test for each aspect reveals that more participants thought that the Kinect version was more fun $(\chi 2=6.3, p=.044)$, more exciting $(\chi 2=7.0, p=.030)$, and more challenging $(\chi 2=16, \mathrm{p} \approx .000)$. They were equally easy to learn as evidenced by a majority of participants choosing neither the mouse nor the Kinect version $(\chi 2=7.0$, $\mathrm{p}=.030)$. There was no difference in the choice of game for frustration $(\chi 2=4.75$, $\mathrm{p}=.093)$. 
Borg Perceived Exertion \% Maximum Heart Rate

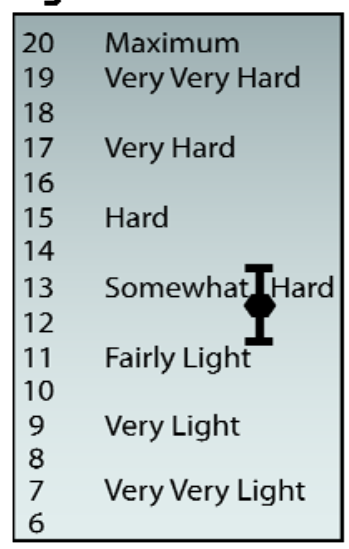

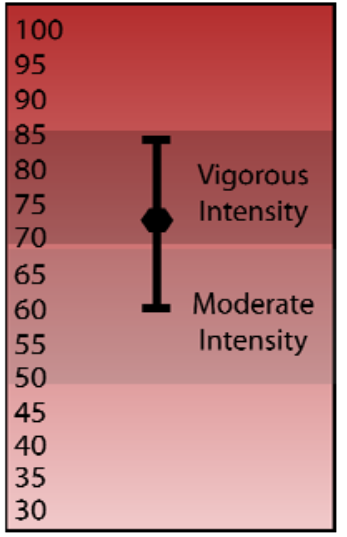

Calories Burned

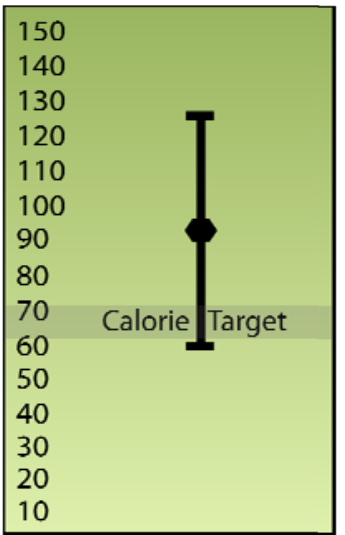

Fig. 3. Our three measures of exercise efficacy. Dots represent the mean of that measurement over all players and error bars represent one standard deviation in player measurements. The average perceived exertion is somewhat hard, the average heart rate is within recommended targets for aerobic activity, and the number of Calories burned exceeds the recommended Calories that adults should burn through physical activity three times daily.

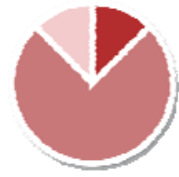

Fun

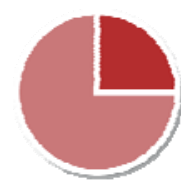

Excting

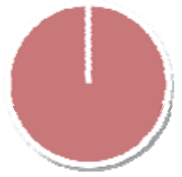

Challenging

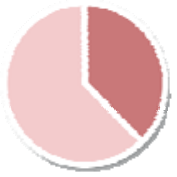

Frustrating

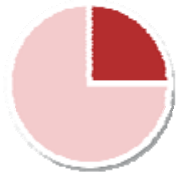

Easy to Leam

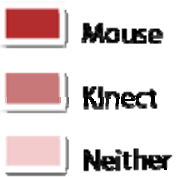

Neither

Fig. 4. Participant rankings for the two versions of the game. The size of the pie wedge for the mouse and Kinect version shows the number of participants who ranked that condition higher. The 'neither' wedge shows the number of participants who ranked both versions equally.

We asked whether participants would consider playing the casual exergame when they had a ten-minute break during their day. Five participants agreed or strongly agreed, with one participant answering each of neutral, disagree or strongly disagree. The participants who disagreed were also two of the bottom three participants in terms of exercise intensity as measured by both target heart rate and Calories burned. It could be that these players were not sufficiently motivated by the game to try hard and would not choose to play it again. It could also be that these players were quite fit and the game did not promote enough activity for them (in which case they don't need a casual exergame to get the recommended daily activity levels). For one of the players, this was the case as s/he already gets over 30 minutes of exercise every day.

\section{Discussion}

Our results showed that GrabApple is fun and that most of our participants would consider playing the casual exergame during breaks in their day. Our results also 
showed that the level of exercise produced by our casual exergame is sufficient to provide health benefits when played a few times per day in 10-minute increments. After playing the Kinect version of GrabApple for ten minutes, participants' average heart rate, Calorie expenditure and Borg exertion rate were all within the recommended exercise intensity [23-25].

In summary, our casual exergame was fun to play and produced a level of exercise consistent with targets for moderate-intensity aerobic activity.

\subsection{Future Work}

Although our initial results are very encouraging, there are three main research directions that we plan to pursue to establish casual exergames as a method for improving health. First, we need to design and implement a suite of casual exergames. To encourage play for the recommended 30 minutes of activity per day, we need to provide players with a selection of games so that repeating the play of a single game during each exercise break does not bore them. As part of this process, we will use the feedback provided by our participants to adjust the gameplay of GrabApple. For example, one player recommended giving score multipliers for consecutive apples grabbed using duck then jump movements. These changes could increase the amount of exercise achieved during play. Second, we need to establish that people are sufficiently motivated by the casual exergames to play them when not participating in an experiment. To find out whether people will partake in short bursts of physical activity by playing casual exergames, we will supply our suite of casual exergames to participants for a longer-term study. Through a combination of data logging and exit interviews, we can establish how well our games motivate people to exercise in small bursts throughout their day. Third, we need to conduct larger studies to establish the health benefits of casual exergames. We plan to consider both physical health and cognitive health as physical activity can relieve symptoms of depression and anxiety [1], and improve mood [1] and cognitive function [2]. Finally, as new forms of activity sensors become available on personal computers, laptops and smartphones, we will consider how to develop casual games for emerging sensing hardware to continue to improve the accessibility of casual games for players.

\section{Conclusion}

To encourage increased physical activity through short bouts of fun activity, we applied the principles of casual game design to exergames in our design and implementation of GrabApple - a casual exergame. Initial tests of GrabApple are encouraging. First most of our participants enjoyed playing the casual exergame and would consider playing the game during breaks in their day. Second, participants' heart rates were elevated to aerobic levels and sufficient Calories were burned during game play. In general, we are encouraged by our preliminary investigation and plan to continue this line of research with further development and formal studies of casual exergames. Casual exergames are an interesting new genre of games that can add physical activity into people's daily routines in an enjoyable way. 
Acknowledgments. We would like to thank NSERC and the GRAND NCE for funding. We would also like to thank members of the HCI lab at the University of Saskatchewan for feedback on the game design and paper, and all of our participants. We particularly thank Kevin Stanley, Nelson Wong, Lennart Nacke and Andre Doucette for their feedback on drafts of the paper and their fine acting in the accompanying video.

\section{References}

1. Physical Activity and Health Executive Summary. U.S. Department of Health and Human Services Centers for Disease Control and Prevention National Center for Chronic Disease Prevention and Health Promotion The President's Council on Physical Fitness and Sports (1999)

2. Hillman, C.H., Erickson, K.I., Kramer, A.F.: Be smart, exercise your heart: exercise effects on brain and cognition. Nat. Rev. Neurosci. 9, 58-65 (2008)

3. Haskell, W.L., Lee, I.-M., Pate, R.R., Powell, K.E., Blair, S.N., Franklin, B.A., Macera, C.A., Heath, G.W., Thompson, P.D., Bauman, A.: Physical Activity and Public Health. Updated Recommendation for Adults From the American College of Sports Medicine and the American Heart Association. Circulation. Circulationaha 107.185649 (2007)

4. Schmidt, W.D., Biwer, C.J., Kalscheuer, L.K.: Effects of Long versus Short Bout Exercise on Fitness and Weight Loss in Overweight Females. J. Am. Coll. Nutr. 20, 494-501 (2001)

5. NASS Spine Health Campaign 2006. North American Spine Society (2006)

6. Mueller, F., Stevens, G., Thorogood, A., O’Brien, S., Wulf, V.: Sports Over a Distance. Personal Ubiquitous Comput. 11, 633-645 (2007)

7. Sinclair, J., Hingston, P., Masek, M.: Considerations for the design of exergames. In: Proceedings of the 5th International Conference on Computer Graphics and Interactive Techniques in Australia and Southeast Asia, pp. 289-295. ACM, New York (2007)

8. Casual Games Market Report 2007. Casual Games Association (2007)

9. Kuittinen, J., Kultima, A., Niemelä, J., Paavilainen, J.: Casual Games Discussion. In: Proceedings of the 2007 Conference on Future Play, pp. 105-112. ACM, New York (2007)

10. Casual Game White Papers I IGDA. International Game Developers Association (2008)

11. Konami Digital Entertainment, Inc., http://www.konami.com/ (Date accessed: April 2011)

12. Nintendo's Wii Video Game Console I Wii Games and Accessories, http://us.wii.com/ (Date accessed: April 2011)

13. Ishii, H., Wisneski, C., Orbanes, J., Chun, B., Paradiso, J.: PingPongPlus: Design of an Athletic-Tangible Interface for Computer-Supported Cooperative Play. In: Proceedings of the SIGCHI Conference on Human Factors in Computing Systems: the CHI is the Limit, pp. 394-401. ACM, New York (1999)

14. Hämäläinen, P., Ilmonen, T., Höysniemi, J., Lindholm, M., Nykänen, A.: Martial Arts in Artificial Reality. In: Proceedings of the SIGCHI Conference on Human Factors in Computing Systems, pp. 781-790. ACM, New York (2005)

15. Stach, T., Graham, T.C.N., Yim, J., Rhodes, R.E.: Heart Rate Control of Exercise Video Games. In: Proceedings of Graphics Interface 2009, pp. 125-132. Canadian Information Processing Society, Toronto (2009) 
16. Yim, J., Graham, T.C.N.: Using Games to Increase Exercise Motivation. In: Proceedings of the 2007 Conference on Future Play, pp. 166-173. ACM, New York (2007)

17. de Oliveira, R., Oliver, N.: TripleBeat: Enhancing Exercise Performance with Persuasion. In: Proceedings of the 10th International Conference on Human Computer Interaction with Mobile Devices and Services, pp. 255-264. ACM, New York (2008)

18. Nenonen, V., Lindblad, A., Häkkinen, V., Laitinen, T., Jouhtio, M., Hämäläinen, P.: Using Heart Rate to Control an Interactive Game. In: Proceedings of the SIGCHI Conference on Human Factors in Computing Systems, pp. 853-856. ACM, New York (2007)

19. Siegel, S.R., Haddock, B.L., Dubois, A.M., Wilkin, L.D.: Active Video/Arcade Games (Exergaming) and Energy Expenditure in College Students. Int. J. Exerc. Sci. 2, 165-174 (2009)

20. Graves, L., Stratton, G., Ridgers, N.D., Cable, N.T.: Comparison of energy expenditure in adolescents when playing new generation and sedentary computer games: cross sectional study. BMJ 335, 1282-1284 (2007)

21. Jakobsson, M., Sotamaa, O.: Game Studies - Special Issue - Game Reward Systems. International Journal of Computer Game Research (2011)

22. Shiffman, D.: Updated Kinect Library for Processing at Daniel Shiffman, http: / / www.shiffman. net/2010/12/18/ updated-kinect-library-for-processing/ (Date accessed: April 2011)

23. Borg, G.: Borg's Perceived Exertion and Pain Scales. Human Kinetics (1998)

24. American College of Sports Medicine: ACSM's Guidelines for Exercise Testing and Prescription. Lippincott Williams \& Wilkins, Philadelphia, Pa (2006)

25. Pate, R.R., Pratt, M., Blair, S.N., Haskell, W.L., Macera, C.A., Bouchard, C., Buchner, D., Ettinger, W., Heath, G.W., King, A.C., Kriska, A., Leon, A.S., Marcus, B.H., Morris, J., Paffenbarger, R.S., Patrick, K., Pollock, M.L., Rippe, J.M., Sallis, J., Wilmore, J.H.: Physical Activity and Public Health: A Recommendation From the Centers for Disease Control and Prevention and the American College of Sports Medicine. JAMA, 402-407 (1995) 